CASE REPORT

\title{
Successful treatment of vitamin D unresponsive hypoparathyroidism with multipulse subcutaneous infusion of teriparatide
}

\author{
Manel Puig-Domingo, Gonzalo Díaz, Joanna Nicolau, Cristián Fernández, Sergio Rueda and Irene Halperin \\ Servei de Endocrinologia, Hospital Clínic, Universitat de Barcelona, O8036 Barcelona, Spain \\ (Correspondence should be addressed to M Puig-Domingo; Email: mpuigd@clinic.ub.es)
}

\begin{abstract}
Objective: Hypoparathyroidism is usually controlled with calcium and vitamin-D supplements; in very few cases this treatment fails and teriparatide may be an alternative. We report the first case of hypoparathyroidism refractory to vitamin-D therapy requiring multipulse teriparatide treatment. Case report: A 53 year-old woman presented severe hypocalcemia and hypomagnesemia after thyroidectomy. Preoperatively, mild hypercalciuria was detected with parathyroid hormone (PTH) $69 \mathrm{pg} / \mathrm{ml}$ (normal 10-45) and 25-OH-vitamin D $9 \mathrm{ng} / \mathrm{ml}$ (normal 20-40) and normal levels of magnesium. No response was seen with oral and i.v. calcium and magnesium, or even with $5 \mu \mathrm{g}$ calcitriol/day, suggesting a vitamin-D resistance status. Calcium sensor and vitamin-D receptor gene mutation studies were negative.

Interventions and results: The following treatments were tried: i) s.c. recombinant human PTH (rhPTH) 1-34 plus oral calcitriol, calcium, and magnesium, was partially effective, but symptoms resumed $4 \mathrm{~h}$ after the injection of $20 \mu \mathrm{g} \mathrm{rhPTH}$; stable calcemia was not achieved even with 4-6 injections/day of teriparatide; ii) two trials of heterologous parathyroid transplant were performed but rejection was detected 3 months after; iii) i.v. magnesium decreased rhPTH requirements but i.m. administration was not tolerated and iv) multipulse s.c. infusion of teriparatide achieved complete normalization of serum calcium, phosphate, magnesium, calciuria and magnesuria with relatively low rhPTH doses (25-35 $\mu \mathrm{g}$ /day) for more than a year.

Conclusions: Vitamin-D unresponsiveness leads to uncontrolled hypocalcemia when postsurgical hypoparathyroidism occurs; in situations of no response to usual or higher doses of vitamin-D and s.c. injections of rhPTH, treatment with teriparatide multipulse s.c. infusor is an effective and safe alternative.
\end{abstract}

European Journal of Endocrinology 159 653-657

\section{Introduction}

Injury or removal of the parathyroid glands during neck surgery is the most common cause of acute and chronic hypoparathyroidism (1). Its incidence rate is usually related to the surgeon's experience, the type of parathyroid pathology and the surgical technique performed. Although postsurgical hypoparathyroidism is usually transitory, it may persist in $0.4-33 \%$ of cases depending on the series $(2,3)$. The conventional treatment of postsurgical chronic hypoparathyroidism is based on calcium salts, vitamin D (mainly calcitriol), and drugs that increase renal tubular reabsorption of calcium as thiazides. Over the past few years, the administration of synthetic recombinant human parathyroid hormone (rhPTH) 1-34 once or twice daily in patients with hypoparathyroidism has proved to reduce urinary calcium excretion compared with calcitriol therapy, and to maintain serum calcium in the normal range, thus avoiding chronic hypercalciuria that may lead to renal function impairment, nephrocalcinosis and renal insufficiency in the long term (4-7).

In the present paper, we describe a 53 year-old woman with post-thyroidectomy uncontrolled hypoparathyroidism despite the usual therapy with high doses of calcitriol plus calcium and magnesium salts. Her life-threatening hypocalcemia and hypomagnesemia required different treatments over 12 months, all of them unsuccessful for controlling calcemia; the final and only effective treatment was a multipulse s.c. infusion of teriparatide which achieved a complete clinical and analytical control of the hypoparathyroid state.

\section{Patients and methods}

\section{Case presentation}

A 53 year-old woman was referred to our Endocrinology Service after thyroidectomy performed due to multinodular goiter with mild retrosternal extension 
and subclinical hyperthyroidism. Prior to this, she had already visited our hospital for a larva migrans chronic infestation acquired during a trip to Thailand 9 years before, and was diagnosed 3 years previously. During the follow-up she developed asthma, and IgG subclasses 1 and 3 deficiencies were detected. During the 8 years, she required corticosteroid treatment either inhaled or oral, sometimes during several months in order to prevent and treat episodes of bronchospasm crisis. Consequently, bone mass was periodically checked; bone mass densitometry (BMD) revealed an osteopenic state; besides this, low 25-hydroxyvitamin D (25-OH-vit D) of $9 \mathrm{ng} / \mathrm{ml}$ (normal 20-40) was also found, together with a moderate increase in circulating PTH level of $69 \mathrm{pg} / \mathrm{ml}$ (normal 10-45), while renal function, calcium (ranging $8.8-9.7 \mathrm{mg} / \mathrm{dl}$ ) and magnesium levels in plasma and urine were normal. These data were interpreted as corticosteroid-induced osteopenia, associated with vitamin D deficiency and some degree of compensatory hyperparathyroidism; she was therefore treated with cholecalciferol over at least 2 years, with normalization of circulating 25-OH-vit D. No bisphosphonate treatment was used at any time. She also suffered two septic episodes during the previous 3 years, one due to enterococcus faecalis and another of polymicrobial ethiology. No septic foci were found despite extensive investigations. Treatment for larva migrans eradication with albendazole, praziquantel, and ivermectine for more than 2 years was ineffective and stopped before treatment of her hyperthyroidism; at present the patient still remains with active infestation.

Total thyroidectomy was performed on April 2005 because of her multinodular goiter, followed by immediate postoperative severe and persistent hypocalcemia. Circulating PTH was undetectable from the first postoperative day. Despite oral calcium and calcitriol started at rapidly progressive doses, serum calcium stayed low (average $6 \mathrm{mg} / \mathrm{dl}$; normal 8.5-10.5) and phosphate high (average $5 \mathrm{meq} / \mathrm{dl}$; normal 2.3-4.3) even with $5 \mu \mathrm{g}$ of calcitriol/day and more than $10 \mathrm{~g}$ calcium/day. She complained of fatigue, palpitations, paresthesia and tetany almost daily, and required emergency assistance with i.v. calcium infusion several times a week because of life threatening hypocalcemia mostly due to severe tetania and impairment of asthma, with increasing frequency of episodes that were more refractory to usual treatment under hypocalcemic conditions (Fig. 1A). Hypocalcemia was associated with low serum magnesium (average $1.5 \mathrm{mg} / \mathrm{dl}$; normal 1.8 2.6), requiring high oral doses plus parenteral loads of magnesium. Urinary excretion of calcium and magnesium markedly increased after i.v. infusion, achieving intolerable high levels, with calciuria up to $900 \mathrm{mg} / 24 \mathrm{~h}$. Despite increasing doses of calcitriol that led to circulating 1,25$\mathrm{OH}$-vitamin $\mathrm{D}$ in the upper range of normality (65 to $75 \mathrm{ng} / \mathrm{l}$; normal $<70 \mathrm{ng} / \mathrm{l}$ ), serum calcium levels remained very low, rarely above $7 \mathrm{mg} / \mathrm{dl}$, apparently indicating some state of vitamin D resistance. A trial of thiazide treatment was unsuccessful with virtually no amelioration of serum calcium levels. A potential malabsorptive state was ruled out by a thorough investigation, including xylose test and radiological and endoscopic procedures, all of which resulted normal. Furthermore, negative antitransglutaminase antibodies ruled out celiac disease. A calcium sensor mutation study was negative. Complete sequencing of the vitamin D receptor gene was performed in Dr Peter Malloy's laboratory at Stanford University as previously described (8) and no mutations were found. After 6 months of uncontrolled life-threatening hypocalcemia and hypomagnesemia under high doses of conventional therapy, the treatments described below were tried after obtaining the patient's informed consent and the approval of the Ethical Committee of the Hospital Clínic Universitari de Barcelona.

\section{Methods}

S.c. rhPTH 1-34 (Forsteo, $20 \mu \mathrm{g} / 80 \mu \mathrm{l}$; Eli Lilly) was started at a dose of $20 \mu \mathrm{g} / 12 \mathrm{~h}$ s.c., according to previously published data $(3,4)$ plus oral calcitriol $5 \mu \mathrm{g} / \mathrm{day}, 10 \mathrm{~g}$ calcium po/day and $0.5 \mathrm{~g}$ magnesium po/day, achieving partial serum calcium control (Fig. 1B); yet, symptoms tended to resume $4 \mathrm{~h}$ after
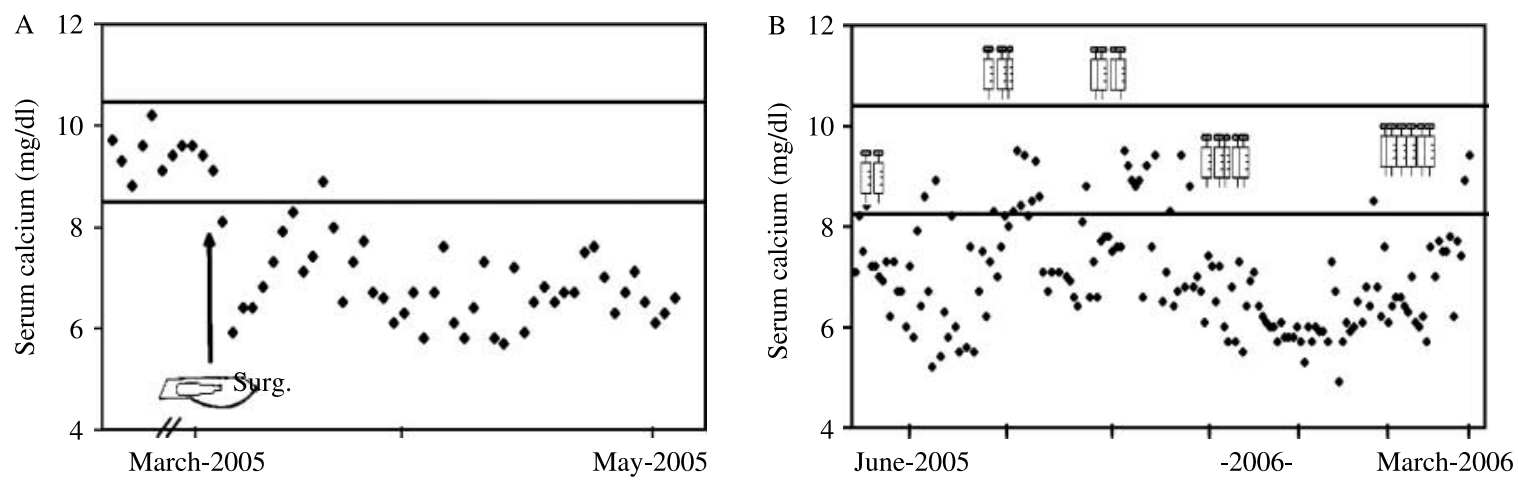

Figure 1 (A) Response to oral calcium up to $10 \mathrm{~g}$ po/day and 1,25-OH-vitamin-D up to $5 \mu \mathrm{g} /$ day after development of hypoparathyroidism following the neck surgery. (B) After 2 months of unresponsiveness rhPTH was added at increasing doses with some initial response but very modest efficacy during 9 months. 
each injection; not even a higher dose frequency (up to 6 injections/day, with a total dose of $120 \mu \mathrm{g} /$ day) was able to achieve stable serum calcium levels.

Heterologous parathyroid transplantation was performed twice. Parathyroid tissue was obtained from a cross-matched patient with end stage renal failure, requiring parathyroidectomy due to tertiary hyperparathyroidism. Multiple portions from an original fragment of $20 \mathrm{mg}$ of the removed hyperplasic parathyroids were implanted in the antebraquial muscle, as previously described (2), under everolimus plus methylprednisolone immunosuppression. Seventy-two hours after the first procedure, PTH was detectable in plasma and a decrease in rhPTH requirement was observed (Fig. 2A). This initial response indicating some viability of the transplanted tissue persisted for 2 weeks, but impairment of calcemic control together with undetectable circulating PTH levels confirmed complete transplant failure 1 month later. A second trial with the same cryopreserved parathyroid tissue was performed 3 months later, but acute rejection was evident 7 days after transplantation despite the addition of mycophenolate to the previous immunosuppressive treatment.

As hypomagnesemia is known to cause a decreased response to PTH action (9), correction of magnesium deficiency was performed by i.v. infusion. The replenishment of magnesium stores and achievement of near normal levels of circulating magnesium resulted in lower rhPTH requirements (4 instead of 6 injections/day, with a total rhPTH requirement that fell from $120 \mu \mathrm{g} /$ day to $80 \mu \mathrm{g}$ /day s.c.; Fig. 2A). However, i.m. magnesium administration was not tolerated due to intense pain at injection site, so this treatment was not feasible under outpatient conditions.

\section{Results}

Multipulse s.c. infusion of teriparatide was started using a MiniMed pump (Medtronic MiniMed, Watford, UK); initially, infusion rate was set up at $60 \mu \mathrm{g} /$ day at the original concentration of $20 \mu \mathrm{g} / 80 \mu \mathrm{l}$ from the Forsteo

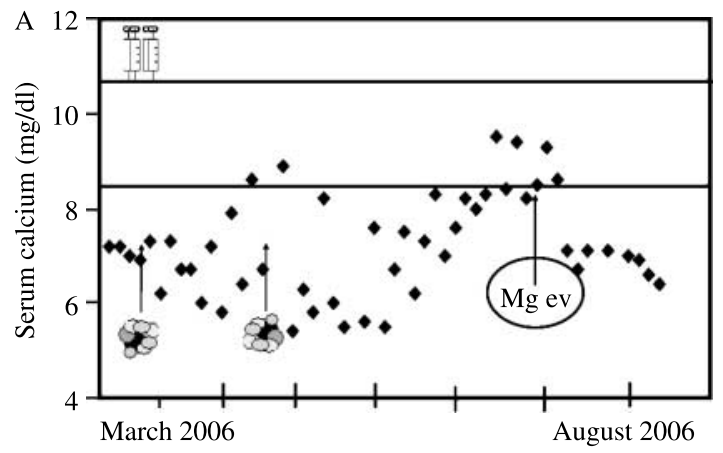

commercial preparation and a complete normalization of serum calcium, phosphate and magnesium, as well as calciuria and magnesuria were achieved in the first day of treatment (Fig. 2B). In the following days, teriparatide infusion rate was tapered down until the minimal dose that allowed a low normal range of calcemia; successful treatment was achieved at final doses of teriparatide ranging between $25-35 \mu \mathrm{g} /$ day. With this treatment regimen serum calcium levels stayed within normal values in most of the follow-up period, until now.

The pump was programmed in order not to give a theoretically continuous delivery of teriparatide, but a multi-micropulse release of the peptide, trying to mimic the physiologic PTH secretion, which has been described to be about 6-7 bursts per hour $(10,11)$. The MiniMed 508 pump used in our patient is based on Bio-Pulse Delivery technology, allowing a pulse design modality of treatment in which a pulse of teriparatide was automatically delivered at intervals of 10-14 min.

The reservoir of the pump was refilled every 3 days with no evidence of a reduction in therapeutic effectiveness over time because of potential peptide degradation due to room temperature exposure. The injection site was carefully cleaned and catheter/needle and injection site changed every 3 days, coinciding with the refilling of the teriparatide reservoir. No major problems have arisen since initiation of this therapeutic modality and the patient has remained virtually normal in serum and urinary calcium and magnesium levels. After 3 months of treatment some loss of efficacy was observed, a tendency toward hypocalcemia, which coincided with the development of micronodules in the s.c. abdominal wall. This episode was rapidly resolved by changing the injection site and not using the specific area for several weeks, during which the micronodularity disappeared spontaneously. On another occasion the patient forgot to refill the reservoir; rapid sub-tetany developed, and the alarms of the infusion system switched on; the episode was resolved with the injection of a bolus of $20 \mu \mathrm{g}$ teriparatide after the reservoir was refilled. Beside these

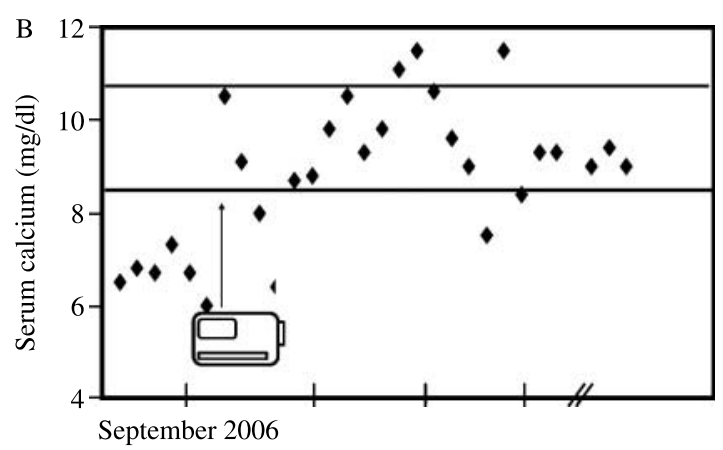

Figure 2 Unsuccessful control of calcemia after two parathyroid transplants; (A) i.v. administration resulted in certain amelioration of calcium levels and a decrease in rhPTH requirements; (B) complete restoration of calcium was achieved after initiation of multipulse s.c. perfusion of teriparatide. 
2 episodes, the patient has remained asymptomatic with normal or near-normal biochemical profile for more than 1 year by using an average of $30 \mu \mathrm{g} /$ day teriparatide; these requirements have remained very stable during this time, indicating a lack of development of anti-teriparatide antibodies or any sort of tachyphylaxia. She did not refer to bone pain and follow-up evaluation of BMD showed a recovery of bone mass, with an increase in $7 \%$ in the first year of treatment, as expected from previously published data (12).

\section{Discussion}

We describe for the first time a case in which teriparatide multipulse pump infusion was the only way to control hypocalcemia in postparathyroidectomy hypoparathyroidism. Fortunately, in most patients with hypoparathyroidism, regardless of the cause, an acceptable control of calcium levels is possible by different dosage regimes of vitamin D and calcium supplements (1). A therapeutic target of low-normal serum calcium, so as to prevent hypercalciuria and long-term detriment of renal function is the ideal. In the clinical setting this is usually feasible, and this is why it is still the treatment of choice of these patients (13). The high cost of rhPTH and the inconvenience of injection treatment are the reasons why vitamin D plus calcium is preferred to this newer, more physiological approach, despite the risk of hypercalciuria. In our patient, we were unable to reestablish circulating normal calcium or at least to avoid symptoms with this usual vitamin $\mathrm{D}$ treatment. Even very high doses of calcitriol $(5 \mu \mathrm{g} /$ day, instead of the recommended 0.25 to $1 \mu \mathrm{g} /$ day), were not effective. A malabsorption state was ruled out by different procedures, and calcitriol oral loads led to normal or higher than normal circulating 1,25-OH-vit D levels together with intense hypercalciuria, indicating that calcium absorption was probably not impaired, although fractional calcium absorption studies were not performed. In order to explain this intriguing lack of effect of very high doses of calcitriol, vitamin D receptor gene was studied (8), but its complete sequencing showed no mutations. As the main regulatory action of vitamin $\mathrm{D}$ is to stimulate calcium absorption in the bowel, hypercalciuria in conjunction with high levels of vitamin D probably indicated that vitamin $\mathrm{D}$ was in fact normally acting at the bowel, while maybe a selective or preferential defect of vitamin $\mathrm{D}$ action on the kidney caused urinary calcium loss leading to hypocalcemia. Alternatively, hypercalciuria may also be due to a specially exquisite dependence of PTH in this patient. Also, no calcium sensor gene mutations were found (14), and although no other potential tubular defects were explored, such as concomitant magnesium, losing disorders or CLDN16 gene mutations $(9,15)$, these possibilities were considered unlikely.
At this point, we tested the hypothesis that the patient was extremely dependent on normal circulating PTH for regulation of her calcium levels. The life threatening condition of her hypocalcemic state, with aggravation of asthmatic crisis, led to transplantation of cross matched parathyroid tissues from end stage renal tertiary hyperparathyroidism patient. This kind of treatment has been used in patients with unresolved hypoparathyroidism despite classic treatment with calcium plus vitamin D (2). The few reported successful cases achieve normal calcium levels about 3 months after the procedure, once the implanted tissue gets vascularized. Rejection may always happen (16), as unfortunately was the case in our patient.

Administration of rhPTH can be an interesting and effective treatment in selective cases such as the present one. In the cohort studied by Winer et al. (4-6), two injections of a mean of $50 \mu \mathrm{g}$ of rhPTH achieved an adequate level of circulating calcium in almost all cases. In our patient such an easy control was not possible, even though doses were swiftly raised from those proposed by Winer, until they reached very frequent peptide injections. We have no explanation for this unresponsiveness to multi-injections of teriparatide. Surprisingly, its administration by a multipulse pump driven system resulted in both highly effective and safe treatment over more than 1 year. Moreover, the absolute dosage used was markedly lower in comparison with the previous ineffective multi-injections schedule, which reduced the cost of teriparatide and was a less uncomfortable treatment for the patient. In the last year a new rhPTH delivery system consisting of an implantable biodegradable polymer has been described, allowing pulsatile release of the peptide, but clinical experience is still lacking (17).

Administration of PTH to animal models has been linked to the development of certain tumors as osteosarcoma (18). A more physiological administration such as the one we used, as the number of pulses delivered per hour in our patient is about the same of normal parathyroid glands $(10,11)$, would probably be safer in this respect; moreover, the total dose given per day in our patient is similar to the one given in the study published by Winer, in which the patients were followed over 3 years without safety problems. A careful follow-up in this regard is provided. In our case no evidence of bone problems were detected after 22 months of teriparatide pump treatment, and additionally, as expected, a recovery of bone mass was observed. We believe that this modality of teriparatide delivery maybe indeed more safe and may allow a potential lower risk of bone problems at long term because of the intermittent nature of the peptide infusion in contrast to a continuous infusion $(19,20)$, although unfortunately we cannot prove that the plasmatic profile of rhPTH in our patient would in fact reproduce the pulse design of the infusion system, as we did not measured circulating PTH in a way to assess it. 
In summary, we report the first case of long term treatment of vitamin D unresponsive hypoparathyroid patient with teriparatide multipulse pump as the only effective therapeutic modality. In our patient this kind of therapy was shown to be safe, as stable profiles of calcium and magnesium were achieved along 1 year, and should therefore be considered in selected cases such as the one described.

\section{Declaration of interest}

The authors declare that there is no conflict of interest that would prejudice their impartiality.

\section{Funding}

This research did not receive any specific grant from any funding agency in the public, commercial or not-for-profit sector.

\section{Acknowledgements}

We are indebted to Dr Peter Malloy at Stanford University for performing the studies of VDR mutations, Dr Ana Aranda from CSIC, Madrid, for case discussion, and Nicki van Berckel for manuscript assistance.

\section{References}

1 Marx SJ. Hyperparathyroid and hypoparathyroid disorders. New England Journal of Medicine 2000343 1863-1875.

2 Torregrosa NM, Rodríguez JM, Llorente S, Balsalobre MD, Rios A, Jimeno L \& Parrilla P. Definitive treatment for persistent hypoparathyroidism in a kidney transplant patient: parathyroid allotransplantation. Thyroid 200515 1299-1302.

3 Page C \& Strunski V. Parathyroid risk in total thyroidectomy for bilateral, benign, multinodular goitre: report of 351 surgical cases. Journal of Laryngology and Otology 2007121 237-241.

4 Winer KK, Ko CW, Reynolds JC, Dowdy K, Keil M, Peterson D, Gerber LH, McGarvey C \& Cutler GB Jr. Long-term treatment of hypoparathyroidism: a randomized controlled study comparing parathyroid hormone-(1-34) versus calcitriol and calcium. Journal of Clinical Endocrinology and Metabolism 200388 4214-4220.

5 Winer KK, Yanovski JA, Sarani B \& Cutler GB Jr. A randomized, cross-over trial of once-daily versus twice-daily parathyroid hormone 1-34 in treatment of hypoparathyroidism. Journal of Clinical Endocrinology and Metabolism 199883 3480-3486.
6 Winer KK, Yanovski JA \& Cutler GB Jr. Synthetic human parathyroid hormone $1-34$ vs calcitriol and calcium in the treatment of hypoparathyroidism. Journal of the American Medical Association 1996276 631-636.

7 Angelopoulos NG, Goula A \& Tolis G. Sporadic hypoparathyroidism treated with teriparatide: a case report and literature review. Experimental and Clinical Endocrinology and Diabetes 2007115 50-54.

8 Malloy PJ, Xu R, Peng L, Peleg S, Al-Ashwal A \& Feldman D. Hereditary 1,25-dihydroxyvitamin $\mathrm{D}$ resistant rickets due to a mutation causing multiple defects in vitamin $\mathrm{D}$ receptor function. Endocrinology 2004145 5106-5114.

9 Konrad M \& Weber S. Recent advances in molecular genetics of hereditary magnesium-losing disorders. Journal of the American Society of Nephrology 200314 249-260.

10 Schaefer F. Pulsatile parathyroid hormone secretion in health and disease. Novartis Foundation Symposium 2000227 225-239.

11 Harms HM, Kaptaina U, Klpmann WR, Brabant G \& Hesch RD. Pulse amplitude and frequency modulation of parathyroid hormone in plasma. Journal of Clinical Endocrinology and Metabolism 198969 843-851.

12 Farooki A, Fornier M \& Girotra M. Anabolic therapies for osteoporosis. New England Journal of Medicine 2007357 2410-2411.

13 Davies M. High-dose vitamin D therapy: indications, benefits and hazards. International Journal for Vitamin and Nutrition Research 198930 81-86.

14 Shiohara M, Shiozawa R, Kurata K, Matsuura H, Arai F, Yasuda T \& Koike K. Effect of parathyroid hormone administration in a patient with severe hypoparathyroidism caused by gain-of-function mutation of calcium-sensing receptor. Endocrine Journal 200653 797-802.

15 Kang JH, Choi HJ, Cho HY, Lee JH, Ha IS, Cheong HI \& Choi Y. Familial hypomagnesemia with hypercalciuria and nephrocalcinosis associated with CLDN16 mutations. Pediatric Nephrology 200520 1490-1493.

16 Nawrot I, Woźniewicz B, Tołoczko T, Sawicki A, Górski A, Chudziński W, Wojtaszek M, Grzesiuk W, Sladowski D, Karwacki J, Zawitkowska T \& Szmidt J. Allotransplantation of cultured parathyroid progenitor cells without immunosuppression: clinical results. Transplantation $2007 \mathbf{2 7} 734-740$.

17 Liu X, Pettway GJ, McCauley LK \& Ma PX. Pulsatile release of parathyroid hormone from an implantable delivery system. Biomaterials 200728 4124-4131.

18 Sato M, Ma YL, Hock JM, Westmore MS, Vahle J, Villanueva A \& Turner CH. Skeletal efficacy with parathyroid hormone in rats was not entirely beneficial with long-term treatment. Journal of Pharmacological and Experimental Therapeutics 2002302 304-313.

19 Poole KE \& Reeve J. Parathyroid hormone - a bone anabolic and catabolic agent. Current Opinion in Pharmacology 20055 612-617.

20 Lotinun S, Sibonga JD \& Turner RT. Differential effects of intermittent and continuous administration of parathyroid hormone on bone histomorphometry and gene expression. Endocrine 200217 29-36.

Received 8 July 2008

Accepted 6 August 2008 